\title{
Nanodiamonds in Oil Emulsions as Effective Vaccine Adjuvants and Antitumor Therapeutic Agents
}

Hsin-Hung Lin

Academia Sinica

Chih-Yen Wang

Academia Sinica

Feng-Jen Hsieh

Academia Sinica

Fang-Zhen Liao

Academia Sinica

Yu-Kai Su

Academia Sinica

Minh Dinh Pham

Vietnam Academy of Science and Technology

Hsao-Hsun Hsu

National Taiwan University Hospital

Huan-Cheng Chang ( $\nabla$ hchang@gate.sinica.edu.tw )

Academia Sinica https://orcid.org/0000-0002-3515-4128

Research

Keywords: Adjuvants, mice, nanodiamonds, ovalbumin, vaccine

Posted Date: January 11th, 2022

DOI: https://doi.org/10.21203/rs.3.rs-707469/v2

License: (c) (i) This work is licensed under a Creative Commons Attribution 4.0 International License.

Read Full License 


\section{Abstract \\ Background}

Composed of mineral oil and mycobacteria pathogens, complete Freund's adjuvant (CFA) is one of the most commonly used adjuvants for antibody production and scientific research due to its high efficiency. However, the dead mycobacteria in CFA can cause many allergic reactions. We propose here a new formulation based on the use of nanodiamonds (NDs) as biocompatible non-allergic additives in incomplete Freund's adjuvant (IFA) to avoid these adverse effects.

\section{Methods}

Chicken egg ovalbumin (OVA) was used as the antigens and 100-nm NDs after purification by air oxidation and strong oxidative acid washes were used as the additives. Levels of OVA-specific IgG antibody in mouse sera were measured by using enzyme-linked immunosorbent assays (ELISA) after the second and third immunizations of healthy mice with OVA and OVA/ND in IFA or CFA. Abilities of the OVA/ND/IFA vaccination to inhibit the tumor growth of mice inoculated with EL4 cells or OVA-expressing E.G7 cells were examined over 1 month.

\section{Results}

The new formulation worked well as a potent vaccine adjuvant, which could boost the immune responses and reduce the consumption of antigens in producing antibodies of interest in model animals like mice. Additionally, the composites showed distinct therapeutic activities, as proven by the OVA/ND/IFA treatment that effectively inhibited the tumor progression of E.G7-inoculated mice, allowing the animals to survive over 35 days post tumor-cell challenges. About $0.2 \%$ of the injected ND particles were found in mouse spleens on day 24 after vaccination of the E.G7-inoculated mice with OVA/ND/IFA.

\section{Conclusions}

The multiple functionality of ND makes it useful as an active and trackable component of a vaccine adjuvant not only to enhance antibody production but also to suppress tumor growth in vivo. The NDbased new formulation can be developed into single-dose vaccines with promising potential for realworld applications.

\section{Introduction}

Vaccination is an effective public health tool to prevent the spread of infectious disease worldwide [1]. It is playing an ever-increasing role in preventing and controlling epidemics (such as COVID-19) today [2]. The use of vaccines is to elicit immunological responses that mediate the protection from infection or 
disease. The majority of vaccine antigens currently available and under development are subunits of pathogens or their recombinant molecules with little or no immunostimulatory activities. However, safety has always been a concern for their applications in humans $[3,4]$. Despite numerous efforts made in the past, only a handful of adjuvants have been included in licensed human vaccines and few are in clinical trials. Among these vaccine adjuvants, alum (or aluminum salt) is the most widely used, although aluminum is a known neurotoxin and our understanding of its toxicology and pharmacokinetics in the human body is still limited $[5,6]$. While new types of adjuvants such as those composed of water-in-oil emulsion, squalene, liposomes, other compounds have been developed, they may have higher local reactogenicity and systemic toxicity than alum alone [7, 8]. How to achieve potent adjuvant effects and yet avoid human reactogenicity or toxicity remains a major challenge in the field of vaccine development to date.

Complete Freund's adjuvant (CFA) is a water-in-oil emulsion containing heat-killed mycobacteria for immunization. It is one of the strongest adjuvants known [9], because the inactivated mycobacteria in CFA effectively attract macrophages and other immune cells to the injection sites, while the oil acts as an insoluble depot of antigens to achieve long-term immunostimulation. These two characteristics together produce a pronounced effect in enhancing the immune responses $[10,11]$. However, the high reactogenicity and toxicity of CFA have precluded its applicability in human vaccination and, as a result, CFA is most commonly used for antibody production in experimental animals. A way to circumvent this limitation is to employ incomplete Freund's adjuvant (IFA), which lacks allergic additives (e.g., the inactivated mycobacteria), and mix it with biocompatible, non-allergic, and non-toxic nanoparticles to reduce the undesirable side effects. Ideal balance of efficacy and safety is possible to be reached by mixing IFA with synthetic nanoparticles that have been developed over the past few decades for vaccination applications $[12,13]$. The approach is appealing in the sense that there have been several completed clinical trials using IFA as vaccine adjuvants to treat diseases like human immunodeficiency virus (HIV) infection and thus is potentially useful in humans [14].

The nanoparticles that have been applied for vaccination can be roughly classified into two types $[12,13]$ : (i) organic nanoparticles including liposomes and polymers; (ii) inorganic nanoparticles including aluminum hydroxides, mesoporous silica, magnetic nanoparticles, gold nanoparticles, and nanodiamonds (NDs). Of particular interest are aluminum hydroxides and mesoporous silica, which have been experimentally demonstrated to be useful as antigen carriers as well as self-adjuvants for vaccine delivery [15-18]. However, the toxic levels of these nanoparticles in the human body are not clear. NDs, in contrast, are known to be chemically inert and have excellent biocompatibility and low cytotoxicity. They have found a wide range of applications in biology and nanomedicine due to their high surface-area-tovolume ratios, versatile surface chemistry, and the capability of emitting near-infrared fluorescence from color centers $[19,20]$. Additionally, NDs have been demonstrated to be able to improve the efficacy of many chemotherapeutic agents by increasing their dispersibility in water, shielding the drug from inactivation, and bypassing the mechanisms of chemoresistance [21-23]. These significant improvements have inspired further research on the sustained and controlled release of therapeutics such as growth factors, peptides, and genes both in vitro and in vivo with the aid of NDs. A recent study has 
shown that NDs can serve as an efficient delivery system for immunostimulatory cytosine-phosphateguanine oligonucleotides with great potential for cancer immunotherapy applications [24]. Another study demonstrated that NDs can be readily taken up by immune cells (including natural killer cells and monocytes), resulting in no compromise in cell viability and immune cell activation, and thus are useful for targeted anti-tumor immunotherapy [25]. Furthermore, fluorescent nanodiamond (FND) particles surface-conjugated with immunomodulatory molecules are promising candidate agents with trackable and traceable capabilities to stimulate and manipulate the immune systems $[26,27]$.

Chicken egg ovalbumin (OVA) was chosen as the model antigen in this study because the protein is a well-characterized target antigen for CD8+ T cells (e.g., cytotoxic T lymphocytes), which specifically recognize the OVA 257-264 peptides and thus offer an excellent opportunity to investigate antigenspecific T cell immunity [28]. The biocompatible, non-allergic, and non-toxic nanoparticles used for this work were monocrystalline NDs of $100 \mathrm{~nm}$ in diameter. Their surfaces were first oxidized in air and subsequently carboxylated by acid treatment to facilitate their conjugation with OVA through electrostatic attraction, hydrogen bonding, van der Waals force, and hydrophobic interactions. The conjugation is expected to help increase the uptake of OVA by antigen-presenting cells (APCs) through endocytosis of NDs and thus enhances their antibody production. To achieve this goal, we first noncovalently conjugated OVA with NDs to form stable complexes and enable the sustained release of these surface-bound immunogens either in vitro or in vivo. We then mixed the antigen-containing buffers with IFA to yield emulsions, followed by subcutaneous injection of the emulsions into healthy mice and monitoring the animals' immune responses. Furthermore, to prove that the OVA-ND complexes can indeed be internalized by APCs, we employed OVA-coated FNDs and provided fluorescence images of these particles taken up by murine macrophages such as RAW264.7 cells. Finally, the immunotherapeutic effects of the vaccine were demonstrated by inoculating tumor-free mice with E.G7-OVA derived from the mouse lymphoma cell line EL4 containing a single copy of an inserted gene for constitutive synthesis and secretion of chicken egg OVA in the cells. How the vaccination inhibited the tumor growth was examined closely over a time period of more than 1 month. A significant enhancement in antibody production and an effective suppression of tumor growth were achieved by using this new ND-IFA-based adjuvant system.

\section{Results And Discussion}

\section{Material characterization}

NDs before and after mixing with OVA were analyzed for their sizes and zeta potentials. Transmission electron microscopy (TEM) of bare NDs after strong oxidative acid washes showed that the particles were irregular in shape and varied considerably in size (inset in Figure 1A). Dynamic light scattering measurements of these acid-treated NDs in distilled deionized water (DDW) revealed a mean hydrodynamic diameter of $\sim 100 \mathrm{~nm}$ with a polydispersity index of 0.12 . The average diameter of the particles after mixing with OVA in DDW increased by about $20 \mathrm{~nm}$, an indication that the NDs have been successfully coated with OVA by physical adsorption. The typical zeta potential of the bare ND particles was $-45 \mathrm{mV}$ (Figure 1A). As the isoelectric point of OVA is 4.5 [29], which means that the protein 
molecules are negatively charged in DDW, the change of the zeta potential from $-45 \mathrm{mV}$ of NDs to -23 $\mathrm{mV}$ of OVA-ND implied that forces (such as hydrophobic forces) other than electrostatic interaction were involved in the protein adsorption processes.

As a member of nanocarbon family, the surface of NDs can be conveniently modified with a variety of oxygen-containing groups such as $-\mathrm{COOH},-\mathrm{COH},-\mathrm{COOC}-$, etc. by extensive washes in strong oxidative acids. Uniquely, the acid-washed NDs exhibit an exceptionally high affinity for a variety of protein molecules, including bovine serum albumin (BSA), myoglobin, cytochrome c, lysozyme, and luciferase [30-32]. Moreover, the structural integrity of these proteins is retained, as demonstrated by the catalytic activities of lysozyme and luciferase after adsorption onto NDs [31,32]. Chicken OVA is a phosphorylated glycoprotein consisting of 385 amino acid residues with a molecular weight of $42.7 \mathrm{kDa}$ or a total molecular weight of $45 \mathrm{kDa}$ (including the carbohydrate and phosphate portions) [29]. To evaluate the amount of OVA that could be loaded on the acid-washed NDs, we measured the changes in absorbance of free OVA at $280 \mathrm{~nm}$ before and after mixing with the nanoparticles, as shown in Figure 1B. For OVA adsorbed on 100-nm NDs, we determined a protein loading capacity of $0.12 \mathrm{~g} / \mathrm{g}$ at saturation. Assuming a spherical shape of the adsorbent, this loading capacity suggests that each 100-nm ND (weight of $\sim 1.8$ $\mathrm{fg} /$ particle) can accommodate more than 3000 OVA molecules on surface.

\section{Antigen uptake}

Prior to the in vivo experiments, we investigated whether or not the OVA-ND conjugates could be readily taken up by APCs in vitro. To address this issue, we chose to use RAW264.7 macrophages as the model cell line $[25,27]$, which was established from tumors induced by Abelson murine leukemia virus in BALB/c mice [33]. Additionally, in order to observe the cellular uptake in real time, we replaced NDs by FNDs that contain a dense ensemble of nitrogen-vacancy centers ( $10 \mathrm{ppm})$ as fluorophores to facilitate their detection by fluorescence imaging [20]. When excited by a green laser, the FNDs produce far-red emission at $700 \mathrm{~nm}$ where the level of cell autofluorescence is low. Moreover, the nitrogen-vacancy centers in the diamond matrix are magneto-optical with unique spin properties, which enable them to be detected with high sensitivity and exceptional selectivity by magnetic modulation [34]. It should be emphasized here that the surface properties of these two types of particles (NDs and FNDs) are essentially identical to each other. They are surface-modified in the same manner (air oxidation and oxidative acid washes), except that the diamond powders used to prepare FNDs are pretreated with electron irradiation and vacuum annealing to create the color centers [35].

Figure 2 presents the confocal fluorescence images of RAW264.7 macrophages after incubation with OVA-FNDs at a particle concentration of $100 \mu \mathrm{g} / \mathrm{mL}$ for $4 \mathrm{~h}$. The images were acquired after removal of the excessive amount of the particles by repeated washes with phosphate-buffered saline (PBS), followed by incubation of the cells in culture medium for more than 3 days. As revealed by the fluorescence images, the OVA-FND conjugates could be spontaneously internalized by the cells in culture medium. Moreover, the cells stayed healthy over $72 \mathrm{~h}$ of the incubation, despite that a large quantity of FNDs were still present inside the cells. The results are in line with that reported by Eidi, et al. [26], who made a comparative study on the cytotoxicity of surface-modified FNDs and alum particles. Cell viability assays 
by the authors using the NSC-34 neuron-like cell line showed that FNDs were non-toxic at the doses of up to $110 \mu \mathrm{g} / \mathrm{mL}$, whereas alum displayed a distinct toxic or severely toxic effect at all doses used over 1 $50 \mu \mathrm{g} / \mathrm{mL}$. The high biocompatibility and photostability of FND makes it useful as a tag for tracking and tracing adjuvant particles in vivo.

Aside from the fluorescence imaging, we further quantified the amount of OVA-FNDs taken up by the macrophages. This was achieved by measuring the fluorescence intensities of the cells dispersed in DDW using the magnetic modulation technique detailed in ref. [34]. It was found that the number of the internalized FNDs scaled nearly linearly with the OVA-FND concentration but gradually leveled off at the particle concentration above $100 \mu \mathrm{g} / \mathrm{mL}$ (Figure 3). For cells incubated with the nanoparticle bioconjugates at $100 \mu \mathrm{g} / \mathrm{mL}$, the average number of the internalized OVA-FNDs was 208. Since each 100nm FND can carry more than 3000 OVA molecules as that of 100-nm NDs, this result suggests that the total number of OVA molecules taken up by the individual RAW264.7 macrophages can exceed $6 \times 10^{5}$. These antigens have a high likelihood to be presented on the surface of the murine macrophages and possibly other APCs as well.

\section{Immune responses}

The in vivo experiments were started by mixing $5 \mu \mathrm{L}$ OVA solution $(1 \mathrm{mg} / \mathrm{mL})$ with $30 \mu \mathrm{L}$ ND suspension $(2 \mathrm{mg} / \mathrm{mL})$, followed by dispersion of the mixtures in CFA or IFA. The corresponding control experiments consisted of $5 \mu \mathrm{g}$ OVA only without NDs. Prior to subcutaneous injection of the ND-based adjuvants into BALB/C mice, it is crucial to know the fraction of OVA attached NDs, which could act as a depot of the antigens, thus promoting their cellular uptake by APCs. UV-Vis spectrophotometric analysis of the OVAND mixture showed that about $40 \%$ of the OVA molecules were attached to NDs and the rest of them were free in solution. They are in dynamic equilibrium with each other. Comparing these values with the protein loading capacity of $0.12 \mathrm{~g} / \mathrm{g}$ as determined earlier suggests a surface coverage of $27 \%$ for OVA on NDs.

Depicted in Figure 4A is the timeline of immunization and blood collection in this experiment. The waterin-oil emulsions formed small nodules and appeared as soft capsules at the injection sites upon immunization. We evaluated the OVA-specific IgG antibody responses with the sera of the immunized mice by using enzyme-linked immunosorbent assays (ELISA) after the second and third immunizations with OVA and OVA/ND in CFA. As shown in Figure 4B and 4C, the OVA/ND/CFA treatments induced a significantly higher amount of OVA-specific IgG antibodies in the mouse sera than OVA/CFA alone (3.5 versus 1.6 folds) after the second and third immunizations. It is demonstrated that the addition of NDs in CFA is able to elicit efficient and protective immune responses against OVA in the mouse body, in good agreement with a previous report with NDs showing enhanced immune responses against recombinant $\mathrm{HA} / \mathrm{H} 7 \mathrm{~N} 9$ in mice [36].

Next, we investigated the dose dependence of the immune response by employing OVA/CFA and OVA/ND/CFA containing $25 \mu \mathrm{g}$ OVA each. The amount of NDs used in these assays increased accordingly to $300 \mu \mathrm{g}$. Indeed, a 2-fold increase of the OVA-specific IgG antibody production was found in 
the OVA/CFA treatment (Figure 4A). However, the response did not exceed that of the $5-\mu \mathrm{g}$ treatment with OVA/ND/CFA. Notably, further increase of the OVA dose failed to boost the immune response in the OVA/ND/CFA treatment. The result pointed toward a saturation effect, where no higher levels of anti-OVA could be reached irrespective of the doses of OVA applied. An important implication of this finding is that the use of NDs as additives in CFA can help reduce the consumption of antigens in producing the antibodies of interest, which is a valuable feature for industrial production of antibodies and vaccines.

We explored further if the same level of immune response by OVA/ND/CFA could be maintained without the need of allergic components such as inactivated mycobacteria in CFA. The dose groups of $5 \mu \mathrm{g}$ OVA were employed in this experiment. As shown in Figure 5, we found no significant differences in the results between the OVA/ND/IFA and OVA/ND/CFA treatments in these groups, indicating that the substitution of dead mycobacteria by NDs as additives in the mineral oil not only can improve the safety but also can maintain the efficacy of the vaccine adjuvant. This new combination of substances is expected to work properly also as immune drug delivery vehicles to promote directed antitumor activities with minimal systemic toxicity [27].

\section{Antitumor therapeutics}

The new formulation of NDs in oil emulsions is applicable as antitumor therapeutic agents as well. We demonstrated the applications by using the mouse lymphoma cell lines, EL4 and E.G7-OVA. The latter cells were derived from the C57BL/6 mouse lymphoma cell line (EL4) transfected with pAc-neo-OVA plasmids [37]. They are able to express OVA and have been widely used in cancer immunotherapy studies. Depicted in Figure 6A is the timeline for the subcutaneous injection of OVA/ND/IFA first, followed by inoculation of EL4 and E.G7-OVA cells in C57BL/ 6 mice. The doses of OVA used in both groups were 5 $\mu \mathrm{g}$. By referring to the unvaccinated groups, we found that the treatment with OVA/ND/IFA in the EL4 model was unable to delay the tumor growth (Figure 6B). In contrast, the OVA/ND/IFA treatment could effectively inhibit the tumor progression in the E.G7 model over 3 weeks post inoculation of the cells (Figure 6C). Notably, half of the mice (4 out of 7 mice) in the E.G7 model maintained their tumor-free status for more than 15 days after cell inoculation (Figure 6D) and survived up to 35 days post tumor cell challenges (Figure 6E). In Figure 6F, we show photographs of the tumors isolated on day 24 from vaccinated and non-vaccinated mice. The difference in tumor size between these two groups (in triplicate) of mice is substantially, about 10 times in total volume.

We next verified the hypothesis that NDs played an important role in the antitumor therapeutic treatment. The verification was made by replacing NDs with FNDs in the adjuvants and searching for the particles in sacrificed mice by fluorescence detection. In this experiment, we followed the same procedures described above for the OVA/ND/IFA treatment and collected the spleen tissues of the mice inoculated with EL4 and E.G7-OVA cells on day 24 after the vaccination with OVA/FND/IFA. With the aid of magnetic modulation to achieve selective detection of FNDs in the tissue digests without any pre-separation [34, 38], we were able to clearly identify the presence of these particles in mouse spleens by measuring the intensities of the far-red fluorescence from nitrogen-vacancy centers at wavelengths longer than $750 \mathrm{~nm}$. The amount 
of FNDs found inside the spleens was $0.13 \mu \mathrm{g}$, obtained after subtraction of the backgrounds from the signals between these two groups (Figure 6G). Given a total weight of $60 \mu \mathrm{g}$ for the FNDs used in vaccination, the recovery rate was estimated to be $0.2 \%$. Taken together, the data displayed in Figure 6 strongly corroborate the suggestion that the presently developed nanovaccines with ND/IFA as adjuvants are promising agents for cancer immunotherapy.

To further assess the therapeutic potential of OVA/ND/IFA, we investigated the in vivo immunostimulatory activity of the agent with just one dose in each mouse. Single-dose therapy has several advantages over multiple-dose therapy, including greater patient compliance, less risk of side effects, and lower costs [39]. In particular, in the efforts of protecting livestock (such as cattle, sheep, pigs, and goats) from infectious diseases, single-dose veterinary vaccine renders it easier for suppliers to streamline the production process and distribution of the agents to rural areas [40]. Furthermore, knowing the effectiveness of the single-dose COVID-19 vaccines composed of either whole viruses, protein subunits, viral vectors, or nucleic acids is crucial in the prevention and control of SARS-CoV-2 infections today [41].

In this single-shot experiment, mice were first administrated with OVA/ND/IFA via subcutaneous injection and then examined by measuring the production of anti-OVA IgG in the mouse sera on a weekly basis. We found that the OVA/ND/IFA treatment could dramatically induce the production of OVA-specific IgG antibodies on day 28 and day 35 after the administration (Figure 7). Compared with the OVA/ND and OVA/IFA groups using the same amount of antigens, the OVA/ND/IFA treatment boosted the levels of anti-OVA IgG by 432 and 6 times on day 28, respectively. The enhancement factor increased to 1717 and 19 times on day 35. It is demonstrated that the addition of NDs can substantially improve the effectiveness of IFA as a single-dose vaccine adjuvant, which is capable of sustaining its immunostimulatory activities over an extended period of time.

Finally, we explored whether or not the addition of NDs in IFA altered the mechanism of the immune response elicited by IFA alone, which is known to proceed predominantly through the Th2 pathway (i.e. humoral immune response) [17, 42]. We addressed the question by performing ELISA assays for cytokines in the sera of C57BL/6 mice after subcutaneous injection with OVA/ND/IFA. As shown in Figure 8 , only a small difference in the interleukin 2 (IL-2) level was found between the control and treatment groups, whereas a marked elevation of the interleukin 4 (IL-4) concentration in the vaccinated group was detected. Together with the results obtained with FNDs as described in the previous sections, we were led to a possible predominant mechanism for the initiation of the immune response by the ND/IFA-based vaccine as follows: (i) formation of nodules with loose structure in mouse tissues after subcutaneous injection of the antigen-loaded ND/IFA emulsion, in which the adjuvants act as a depot; (ii) sustained release of the antigens from NDs in the water phase of the emulsions; (iii) active and continuous recruitment of immature immune cells to the depot; (iv) uptake of the antigen-loaded NDs by the immune cells through endocytosis; and (v) promotion of Th2 response, where helper T cells bind with the antigen presenting cells and activate the development of B cells into antibody-producing plasma cells in spleens. The proposed mechanism is depicted in Figure 9. 


\section{Conclusion}

We have demonstrated a new formulation, consisting of a ND suspension mixed with IFA, that is capable of generating effective and durable immune responses in mice. Without the need of allergic additives (i.e. dead mycobacteria), the addition of highly biocompatible NDs (diameter of $\sim 100 \mathrm{~nm}$ ) in the oil emulsion can not only retain the adjuvanticity of IFA but also significantly reduce the level of side effects.

Compared with existing products, the ND-in-oil adjuvant has three major advantages: high safety, low side effects, and low demand in antigen. By applying OVA as a model antigen in small animals like mice, our studies show that ND/IFA can serve well as an active vaccine platform to induce sustained and potent immune responses. Additionally, the adjuvants are useful as antitumor therapeutic agents, as proven by the OVA/ND/IFA treatment which effectively inhibits the tumor progression of OVA-expressing E.G7 cells inoculated onto mice. Furthermore, the agents are trackable and traceable in vivo if NDs are replaced by FNDs. It is anticipated that further research, development, and optimization of the ND-based new formulation into single-dose vaccines will enable real-world applications of this technology in diverse areas, particularly in the care and protection of domesticated animals.

\section{Experimental Section}

\section{Chemicals and reagents}

OVA, CFA, IFA, PBS, oxidative acids, and all other chemicals were from MilliporeSigma and used without further purification. Mouse OVA-specific IgG antibodies were obtained from Abcam.

\section{NDs and OVA-NDs}

Monocrystalline synthetic diamond powders with a nominal size of $100 \mathrm{~nm}$ were obtained from Element Six. To remove metallic impurities and graphitic carbon atoms on the surface, the diamond powders were first oxidized in air at $490^{\circ} \mathrm{C}$ for $2 \mathrm{~h}$, followed by microwave cleaning in concentrated $\mathrm{H}_{2} \mathrm{SO}_{4}-\mathrm{HNO}_{3}(3: 1$, $\mathrm{v} / \mathrm{v}$ ) solution at $100^{\circ} \mathrm{C}$ for $3 \mathrm{~h}$ to functionalize their surfaces with $-\mathrm{COOH}$ groups [31]. The NDs were then non-covalently conjugated with OVA via a simple mixing of $5 \mu \mathrm{L}$ OVA solution ( $1 \mathrm{or} 5 \mathrm{mg} / \mathrm{mL}$ ) with $30 \mu \mathrm{L}$ ND suspension ( 2 or $10 \mathrm{mg} / \mathrm{mL}$ ) by shaking for $1 \mathrm{~h}$ at room temperature.

\section{Particle characterization}

Hydrodynamic sizes and zeta potentials of bare NDs and OVA-conjugated NDs were measured with a particle size and zeta potential analyzer (DelsaNano C, Beckman Coulter). The morphologies of NDs deposited on copper grids were imaged with a transmission electron microscope ( $\mathrm{H}-7650$, Hitachi) operating at an acceleration voltage of $75 \mathrm{kV}$.

\section{Protein adsorption}

A UV-Vis spectrophotometer (U-3310, Hitachi) measured the relative amounts of OVA in solution and on ND surface in the OVA-ND mixtures. To carry out the measurements, absorption spectra of OVA with known concentrations (i.e. $100 \mu \mathrm{g} / \mathrm{mL}$ ) were first acquired with the spectrophotometer. The OVA-ND 
mixtures were then centrifuged at $20,000 \times g$ for $15 \mathrm{~min}$ to precipitate NDs. The amounts of OVA remaining in the supernatants were finally quantified by measuring their absorption strengths at $280 \mathrm{~nm}$.

\section{FNDs and OVA-FNDs}

Nitrogen-vacancy centers in NDs were produced by radiation-damage of monocrystalline diamond powders ( $100 \mathrm{~nm}$ in diameter) with a $10-\mathrm{MeV}$ electron beam, followed by vacuum annealing at $800^{\circ} \mathrm{C}$ for $2 \mathrm{~h}$, as previously described [35]. The as-produced FNDs were then treated by air-oxidation and strong acid washes as that of NDs, and finally conjugated with OVA by physical adsorption.

\section{Antigen uptake}

RAW264.7 cells were obtained from Bioresource Collection and Research Center, Taiwan. They were first seeded at a density of $2 \times 10^{5}$ cells per $35-\mathrm{mm}$ dish and then maintained in regular Dulbecco's modified Eagle's medium (DMEM) supplemented with $10 \%$ fetal bovine serum and new born calf serum (Biological Industries) at $37^{\circ} \mathrm{C}$ with $5 \% \mathrm{CO}_{2}$ in a humidified incubator. Afterwards, the cells were incubated with OVAcoated FNDs in serum-free DMEM with concentrations in the range of $0-200 \mu \mathrm{g} / \mathrm{mL}$ at $37^{\circ} \mathrm{C}$ for $4 \mathrm{~h}$ to facilitate their cellular uptake. Confocal fluorescence imaging was conducted after removal of free OVAFNDs not internalized by the cells.

\section{Confocal fluorescence microscopy}

Optical images were acquired with a laser-scanning confocal fluorescence microscope (SP-8, Leica) equipped with a supercontinuum white-light laser operating at $561 \mathrm{~nm}$ for the excitation of FNDs. The corresponding fluorescence was collected through an oil-immersion objective (63x, NA 1.4) and detected at the emission wavelength of $650-800 \mathrm{~nm}$. Internalization of the FND particles by cells were confirmed by three-dimensional imaging.

\section{FND quantification}

The amounts of OVA-FNDs taken up by RAW264.7 cells were measured by using a home-built magnetically modulated fluorescence spectrometer as detailed in ref. [34]. Prior to the measurements, the FND-internalized cells after detachment by trypsinization were sonicated in PBS for $1 \mathrm{~h}$ to release the internalized particles into solution. Fluorescence intensities were then measured directly for the FND suspensions in glass test tubes by laser excitation at $532 \mathrm{~nm}$ through magnetic modulation to achieve background-free detection. The same technique was applied to quantify the amounts of FNDs in mouse spleens. Specifically, in this experiment, mouse spleens $(0.1 \mathrm{~g} /$ each, wet weight) were digested in concentrated $\mathrm{HNO}_{3}(1 \mathrm{~mL})$ at $120^{\circ} \mathrm{C}$ for several hours until the solution became clear. No pre-separation steps of the FND particles were required in the fluorescence intensity measurements.

\section{Cell cultures}

EL4 and E.G7-OVA cells were obtained from Bioresource Collection and Research Center, Taiwan, and used together with the mouse models. The EL4 cells were grown in DMEM culture medium (Thermo Fisher Scientific) complemented with 10\% horse serum and Antibiotic-Antimycotic, whereas the E.G7-OVA 
cells were maintained in RPMI-1640 medium (Thermo Fisher Scientific) supplemented with $10 \%$ fetal bovine serum, $10 \mathrm{mM}$ HEPES, $1.0 \mathrm{mM}$ sodium pyruvate and supplemented with $0.05 \mathrm{mM} 2-$ mercaptoethanol and $0.4 \mathrm{mg} / \mathrm{mL} \mathrm{G} 418$ at $37^{\circ} \mathrm{C}$ in a humidified atmosphere of $5 \% \mathrm{CO}_{2}$

\section{Animals}

BALB/C mice and C57BL/6 mice (female, 6 - 8 weeks) were obtained from BioLASCO, Taiwan.

\section{Immunization}

Mice were immunized by subcutaneous injection of $100 \mu \mathrm{L}$ solutions or emulsions containing either (i) OVA in PBS, (ii) OVA in CFA, (iii) OVA in IFA, (iv) OVA/ND in PBS, (v) OVA/ND in CFA, or (vi) OVA/ND in IFA on day 1,14 , and 28 . The water-in-oil emulsions were prepared by mixing $35 \mu \mathrm{L}$ of OVA/ND suspensions with $65 \mu \mathrm{L}$ of CFA or IFA. The immunogens and the adjuvants were thoroughly emulsified by repeatedly pipetting up and down before injection.

\section{Antitumor therapeutics}

C57BL/6 mice were subcutaneously immunized with the vaccine formulation as solutions or emulsions for 3 times at 2-week intervals and then challenged by E.G7-OVA lymphoma cells ( $5 \times 10^{5}$ cells) or OVAnegative EL4 tumor cells $\left(5 \times 10^{5}\right.$ cells) on day 7 post the final immunization. The tumor growth was monitored starting from day 10 post tumor cell inoculation. For the single-dose therapeutic treatments, anti-OVA IgG was examined on a weekly basis after the immunization.

\section{IgG antibody assays}

Mouse blood was collected from the submandibular veins of vaccinated or non-vaccinated mice on various days after immunization. OVA-specific IgG antibody responses of the immunized mice were evaluated by using ELISA with the collected mouse sera measured in a microplate reader (GloMax, Promega).

\section{Declarations}

\section{Ethics approval and consent to participate}

All the procedures related to animal experiments were carried out in bioassay laboratories and the animal studies were approved by the Institutional Animal Care and Use Committee of the National Taiwan University College of Medicine (NTUCM).

\section{Consent for publication}

Not applicable.

\section{Availability of data and material}

All the data are available on request from the corresponding authors. 


\section{Competing interests}

The authors declare no competing interests.

\section{Authors' contributions}

H.H.L. and Y.Y.H. performed the animal experiments, analyzed the data, and wrote the manuscript, F.J.H., F.Z.L., and Y.K.S. prepared the samples and characterized the materials, M.D.P., H.H.H., and H.C.C. wrote and edited the manuscript. All authors reviewed the manuscript.

\section{Acknowledgements}

This work was supported by Academia Sinica, National Taiwan University College of Medicine, and the Ministry of Science and Technology, Taiwan, under the Grant Nos. MS441, 109-S4672, and 109-2923-M001-011-MY3. We thank Pao Chi Wang for drawing the illustration in Figure 9.

\section{References}

1. B. Greenwood, The contribution of vaccination to global health: past, present and future. Philos. Trans. R. Soc. Lond. B Biol. Sci. 2014, 369, 20130433.

2. W. J. Wiersinga, A. Rhodes, A. C. Cheng, S. J. Peacock, H. C. Prescott, Pathophysiology, transmission, diagnosis, and treatment of coronavirus disease 2019 (COVID-19). JAMA2020, 324, 782.

3. A. M. Harandi, G. Davies, O. F. Olesen, Vaccine adjuvants: scientific challenges and strategic initiatives. Expert Rev. Vaccines2009, 8, 293.

4. A. Batista-Duharte, E. B. Lindblad, E. Oviedo-Orta, Progress in understanding adjuvant immunotoxicity mechanisms. Toxicol. Lett. 2011, 203, 97.

5. L. Tomljenovic, C. A. Shaw, Aluminum vaccine adjuvants: are they safe? Curr. Med. Chem. 2011, 18, 2630.

6. T. R. Ghimire, The mechanisms of action of vaccines containing aluminum adjuvants: an in vitro vs in vivo paradigm. SpringerPlus 2015, 4, 181.

7. N. Petrovsky, S. Heinzel, Y. Honda, A. B. Lyons, New-age adjuvants: friend or foe? Biopharm. Internationa/2007, 20, A24.

8. N. Petrovsky, Comparative safety of vaccine adjuvants: A summary of current evidence and future needs. Drug Saf. 2015, 38, 1059.

9. S. Gallucci, M. Lolkema, P. Matzinger, Natural adjuvants: Endogenous activators of dendritic cells. Nat. Med. 1999, 5, 1249.

10. H. F. Stils, Jr. Adjuvants and antibody production: Dispelling the myths associated with Freund's complete and other adjuvants, ILAR J.2005, 46, 280.

11. S. Awate, L. A. Babiuk, G. Mutwiri, Mechanisms of action of adjuvants, Front. Immunol. 2013, 4, 114. 
12. L. Zhao, A. Seth, N. Wibowo, C. X. Zhao, N. Mitter, C. Yu, A. P. Middelberg, Nanoparticle vaccines. Vaccine2014, 32, 327.

13. P. Lung, J. Yang, Q. Li, Nanoparticle formulated vaccines: opportunities and challenges, Nanoscale2020, 12, 5746.

14. J. S. Apostólico, V. A. S. Lunardelli, F. C. Coirada, S. B. Boscardin, D. S. Rosa, Adjuvants: classification, modus operandi, and licensing, J. Immunol. Res. 2016, 2016, 1459394.

15. P. He, Y. Zou, Z. Hu, Advances in aluminum hydroxide-based adjuvant research and its mechanism. Hum. Vaccin. Immunother. 2015, 11, 477.

16. X. Li, A. M. Aldayel, Z. Cui, Aluminum hydroxide nanoparticles show a stronger vaccine adjuvant activity than traditional aluminum hydroxide microparticles. J. Control Release2014, 173, 148.

17. K. T. Mody, A. Popat, D. Mahony, A. S. Cavallaro, C. Yu, N. Mitter, Mesoporous silica nanoparticles as antigen carriers and adjuvants for vaccine delivery. Nanoscale2013, 5, 5167.

18. D. Mahony 1, A. S Cavallaro, F. Stahr, T. J Mahony, S. Z. Qiao, N. Mitter, Mesoporous silica nanoparticles act as a self-adjuvant for ovalbumin model antigen in mice. Small 2013, 9, 3138.

19. D. Ho, ed. Nanodiamonds: Applications in Biology and Nanoscale Medicine, Springer, 2009.

20. H.-C. Chang, W. W.-W. Hsiao, M.-C. Su, Fluorescent Nanodiamonds, Wiley, 2018.

21. E. K. Chow, X.-Q. Zhang, M. Chen, R. Lam, E. Robinson, H. Huang, D. Schaffer, E. Osawa, A. Goga, D. Ho, Nanodiamond therapeutic delivery agents mediate enhanced chemoresistant tumor treatment. Sci. Transl. Med.2011, 3, 73ra21.

22. L. Moore, E.K.-H. Chow, E. Osawa, J.M. Bishop, D. Ho, Diamond-lipid hybrids enhance chemotherapeutic tolerance and mediate tumor regression. Adv. Mater.2013, 25, 3532.

23. G. Xi, E. Robinson, B. Mania-Farnell, E. F. Vanin, K.-W. Shim, T. Takao, E.V. Allender, C. S. Mayanil, M. B. Soares, D. Ho, T. Tomita, Convection-enhanced delivery of nanodiamond drug delivery platforms for intracranial tumor treatment. Nanomedicine 2014, 10, 381.

24. Y. Zhang, Z. Cui, H. Kong, K. Xia, L. Pan, J. Li, Y. Sun, J. Shi, L. Wang, Y. Zhu, C. Fan, One-shot immunomodulatory nanodiamond agents for cancer immunotherapy. Adv. Mater. 2016, $28,2699$.

25. L. P. Suarez-Kelly, A. R. Campbell, I. V. Rampersaud, A. Bumb, M. S. Wang, J. P. Butchar, S. Tridandapani, L. Yu, A. A. Rampersaud, W. E. Carson, Fluorescent nanodiamonds engage innate immune effector cells: A potential vehicle for targeted anti-tumor immunotherapy. Nanomedicine2017, 13, 909.

26. H. Eidi, M.-O. David, G. Crépeaux, L. Henry, V. Joshi, M.-H. Berger, M. Sennour, J. Cadusseau, R. K. Gherardi, P. A. Curmi, Fluorescent nanodiamonds as a relevant tag for the assessment of alum adjuvant particle biodisposition. BMC Med. 2015, 13, 144.

27. L. P. Suarez-Kelly, S. H. Sun, C. Ren, I. V. Rampersaud, D. Albertson, M. C. Duggan, T. C. Noel, N. Courtney, N. J. Buteyn, C. Moritz, L. Yu, V. O. Yildiz, J. P. Butchar, S. Tridandapani, A. A. Rampersaud, W. E. Carson, Antibody conjugation of fluorescent nanodiamonds for targeted innate immune cell activation, ACS Appl. Nano Mater. 2021, 4, 3122. 
28. G. B. Lipford, M. Hoffman, H. Wagner, K. Heeg, Primary in vivo responses to ovalbumin. Probing the predictive value of the Kb binding motif. J. Immunol. 1993, 150, 1212.

29. E. Li-Chan, S. Nakai, Biochemical basis for the properties of egg white, Crit. Rev. Poult. Biol.1989, 2, 21.

30. X. L. Kong, L. C. L. Huang, C.-M. Hsu, W.-H. Chen, C.-C. Han, H.-C. Chang, High-affinity capture of proteins by diamond nanoparticles for mass spectrometric analysis, Anal. Chem.2005, 77, 259.

31. T. T. B. Nguyen, H.-C. Chang, V. W.-K. Wu, Adsorption and hydrolytic activity of lysozyme on diamond nanocrystallites, Diamond Relat. Mater.2007, 16, 872.

32. L.-J. Su, H.-H. Lin, M.-H. Wu, L. Pan, K. Yadav, H.-H. Hsu, T.-Y. Ling, Y.-T. Chen, H.-C. Chang, Intracellular delivery of luciferase with fluorescent nanodiamonds for dual-modality imaging of human stem cells, Bioconjugate Chem.2019, 30, 2228.

33. RAW 264.7 (ATTC ${ }^{\circledR}$ TIB-71 ${ }^{\text {TM }}$ ) https://www.atcc.org/products/tib-71

34. Y. Y. Hui, O. Y. Chen, H.-H. Lin, Y.-K. Su, K. Chen, C.-Y. Wang, W. W.-W. Hsiao, H.-C. Chang, Magnetically modulated fluorescence of nitrogen-vacancy centers in nanodiamonds for ultrasensitive biomedical analysis, Anal. Chem. 2021, 93, 7140.

35. H.-C. Lu, Y.-C. Peng, S.-L. Chou, J.-I. Lo, B.-M. Cheng, and H.-C. Chang, Far-UV excited luminescence of nitrogen-vacancy centers: Evidence for diamonds in space, Angew. Chem. Int. Ed.2017, 56, 14469.

36. N. B. Pham, T. T. Ho, G. T. Nguyen, T. T. Le, N. T. Le, H.-C. Chang, M. D. Pham, U. Conrad, H. H. Chu, Nanodiamond enhances immune responses in mice against recombinant HA/H7N9 protein, $J$. Nanobiotechnol.2017, 15, 69.

37. E.G7-OVA [derivative of EL4] (ATCC $®$ CRL-2113 ${ }^{\text {TM }}$ ) https://www.atcc.org/Products/All/CRL2113.aspx\#characteristics

38. L.-J. Su, M.-S. Wu, Y. Y. Hui, B.-M. Chang, L. Pan, P.-C. Hsu, Y.-T. Chen, H.-N. Ho, Y.-H. Huang, T.-Y. Ling, H.-H. Hsu, and H.-C. Chang, Fluorescent nanodiamonds enable quantitative tracking of human mesenchymal stem cells in miniature pigs, Sci. Rep.2017, 7, 45607.

39. I. W. Fong, Single-dose therapy of infectious diseases, Can. Fam. Physician1987, 33, 1239.

40. G. Lofano, C. P. Mallett, S. Bertholet, D. T. O’Hagan, Technological approaches to streamline vaccination schedules, progressing towards single-dose vaccines, npj Vaccines 2020, 5, 88.

41. A. D. Paltiel, A. Zheng, J. L. Schwartz, Speed versus efficacy: quantifying potential tradeoffs in COVID-19 vaccine deployment, Ann. Intern. Med.2021, M20-7866.

42. N. Petrovsky, J. C. Aguilar, Vaccine adjuvants: Current state and future trends. Immunol. Cell Biol. 2004, 82, 488.

\section{Figures}



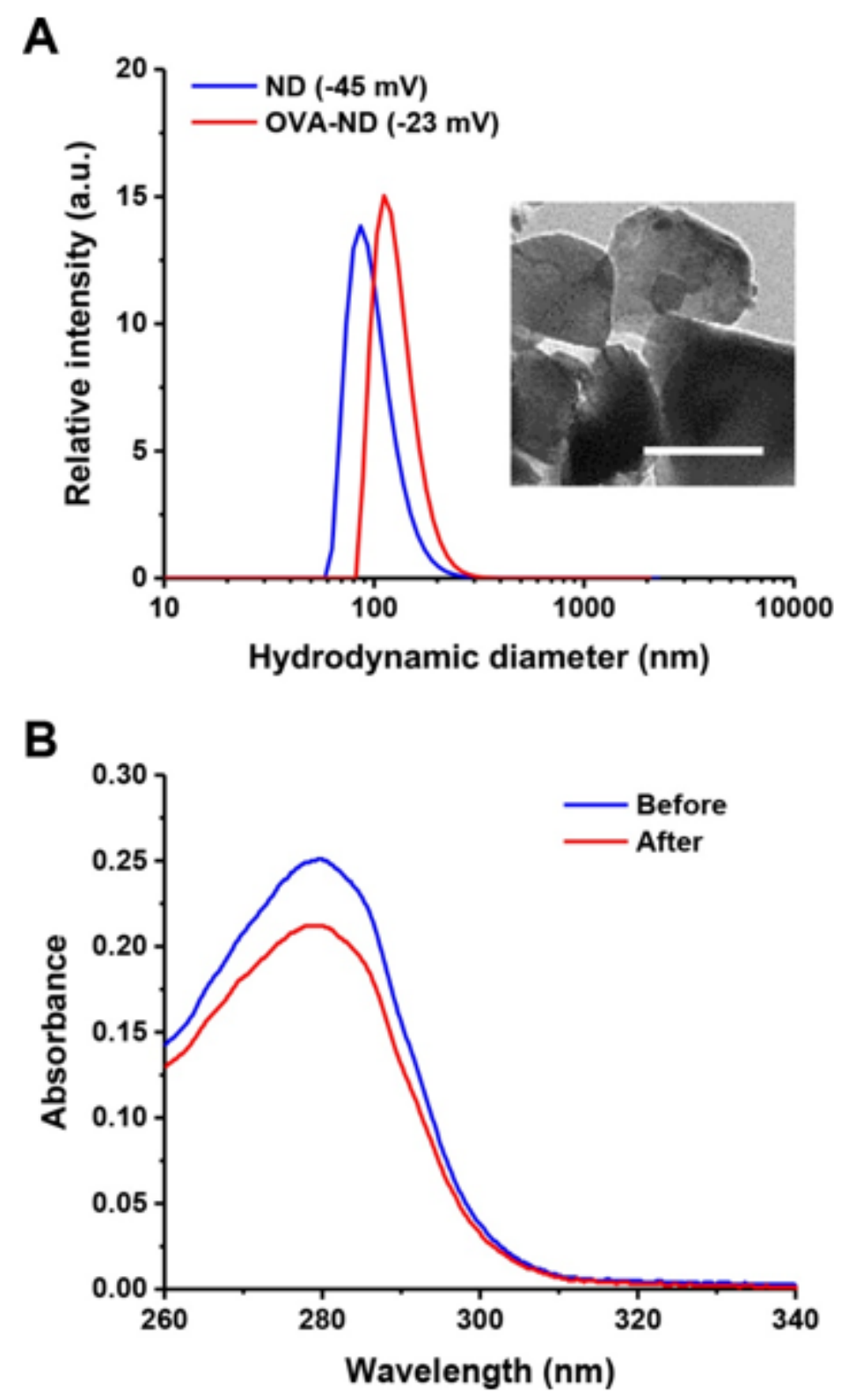

Figure 1

(A) Hydrodynamic sizes of bare NDs and OVA-conjugated NDs in DDW. Values given in the parentheses of annotations are zeta potentials. Inset: TEM image of bare NDs. Scale bar: $100 \mathrm{~nm}$. (B) UV-Vis spectrophotometric analysis of OVA attachment to 100-nm NDs. The absorption spectra were acquired for free OVA before and after interactions with acid-treated NDs in DDW under the conditions of OVA (800 $\mu \mathrm{g} / \mathrm{mL})$ and $\mathrm{ND}(1 \mathrm{mg} / \mathrm{mL})$ with a volume ratio of $1: 1$. The change in absorbance at $280 \mathrm{~nm}$ clearly indicates the adsorption of OVA onto NDs. 


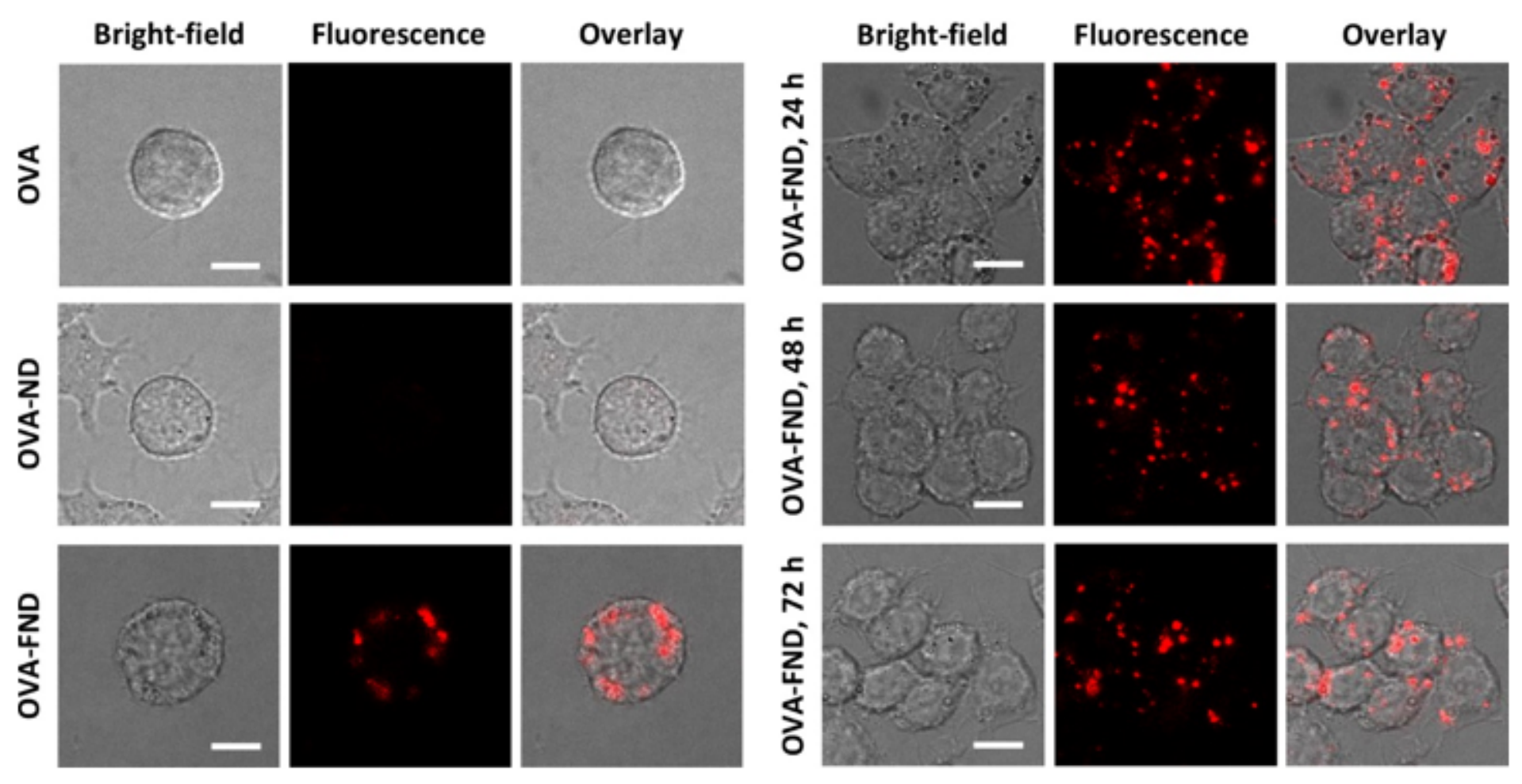

Figure 2

Confocal fluorescence images of RAW264.7 cells with OVA-conjugated FNDs internalized in the cytosol. The cells were incubated with the particles at a concentration of $100 \mu \mathrm{g} / \mathrm{mL}$ for $4 \mathrm{~h}$, after which the excessive amount of OVA-FNDs were removed by PBS washes. Revealed by the images shown to the right, the cells stayed healthy over $72 \mathrm{~h}$ after the particle internalization. Scale bars: $10 \mu \mathrm{m}$.

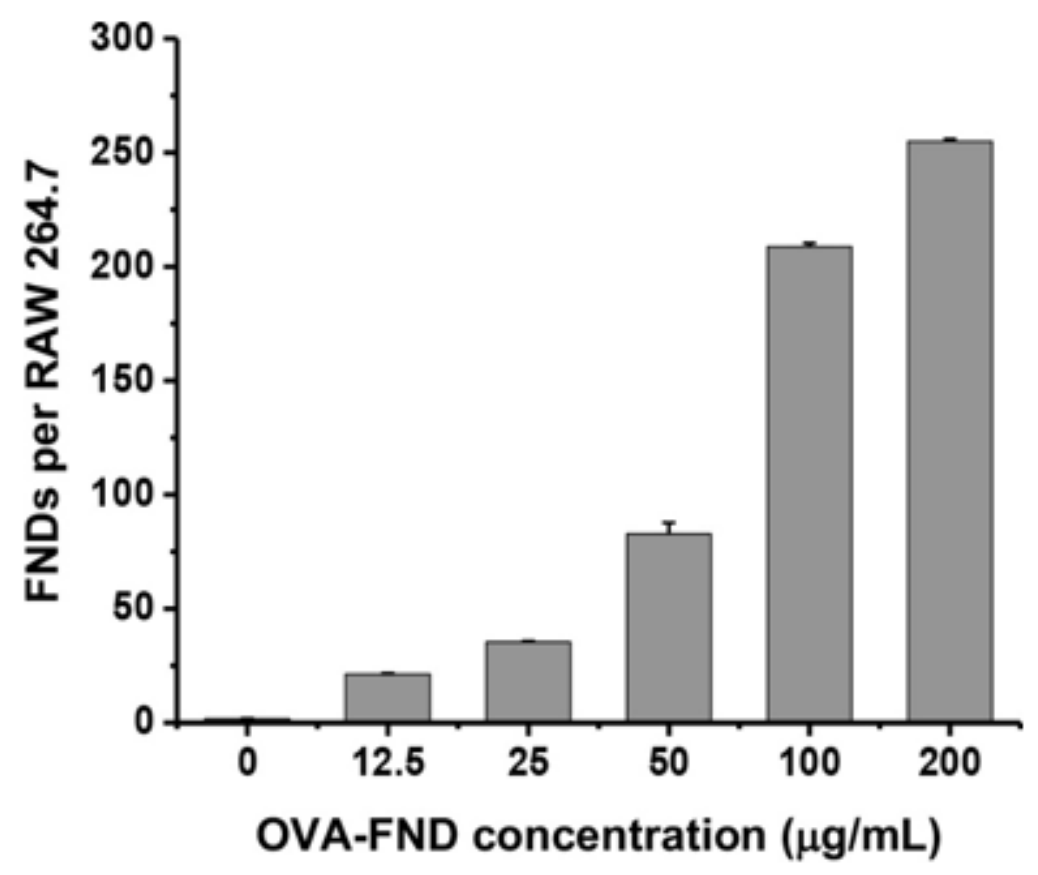

Figure 3 
Amounts of OVA-FNDs internalized by RAW264.7 cells as a function of the particle concentration over 0 $200 \mu \mathrm{g} / \mathrm{mL}$.

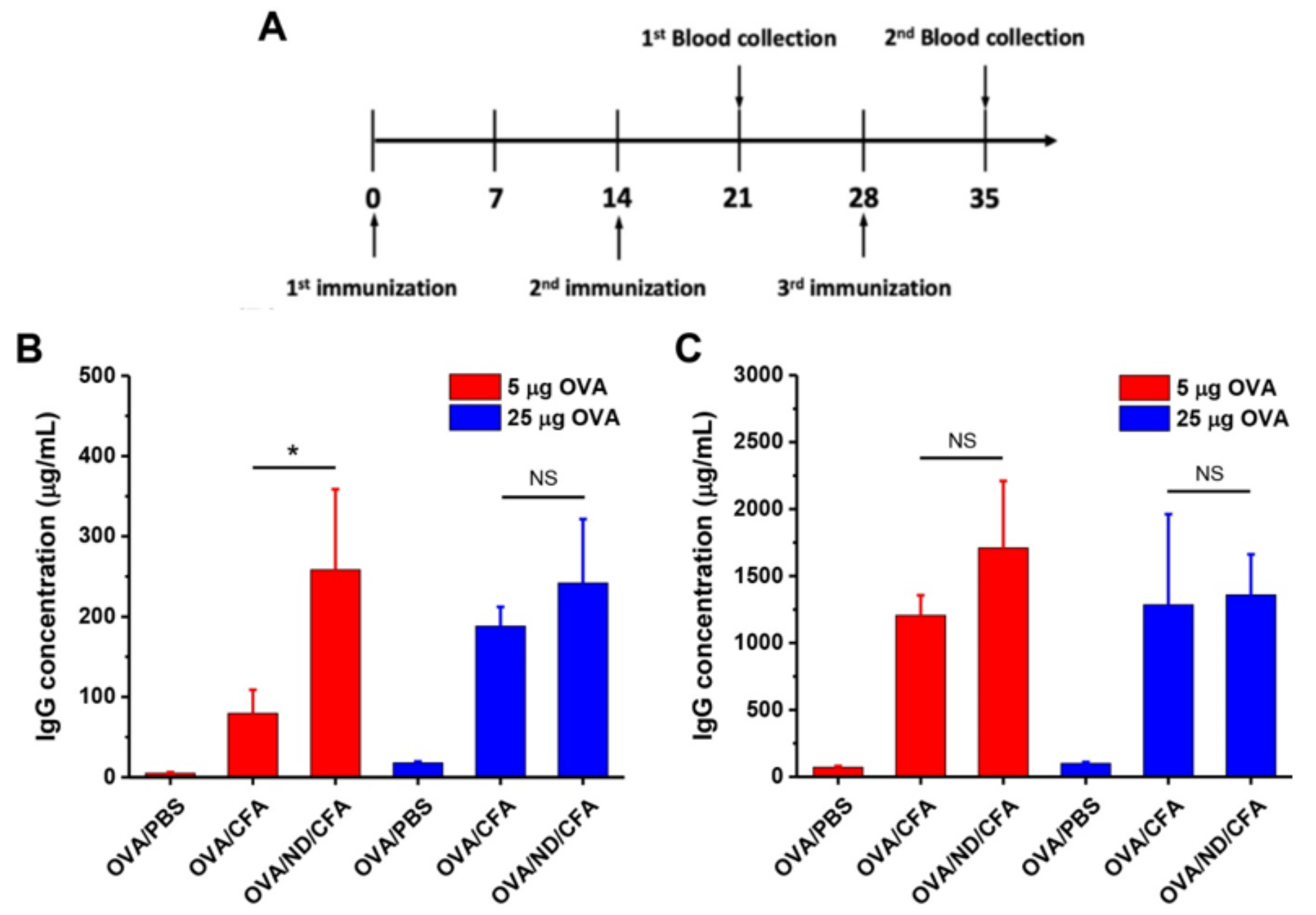

Figure 4

(A) Timeline of the experimental procedures using OVA/ND/CFA for anti-OVA production in mice. (B) Concentrations of OVA-specific IgG antibodies in mouse sera after subcutaneous injection of $5 \mu \mathrm{g}$ and 25 $\mu \mathrm{g}$ OVA along with their adjuvants in mice after the second immunization. (C) Concentrations of OVAspecific IgG antibodies in mouse sera after subcutaneous injection of $5 \mu \mathrm{g}$ and $25 \mu \mathrm{g}$ OVA along with their adjuvants in mice after the third immunization. Values in $(B)$ and $(C)$ are means \pm standard deviations for 3 mice/group ( ${ }^{\star} p \leq 0.05$, NS: not significant). 


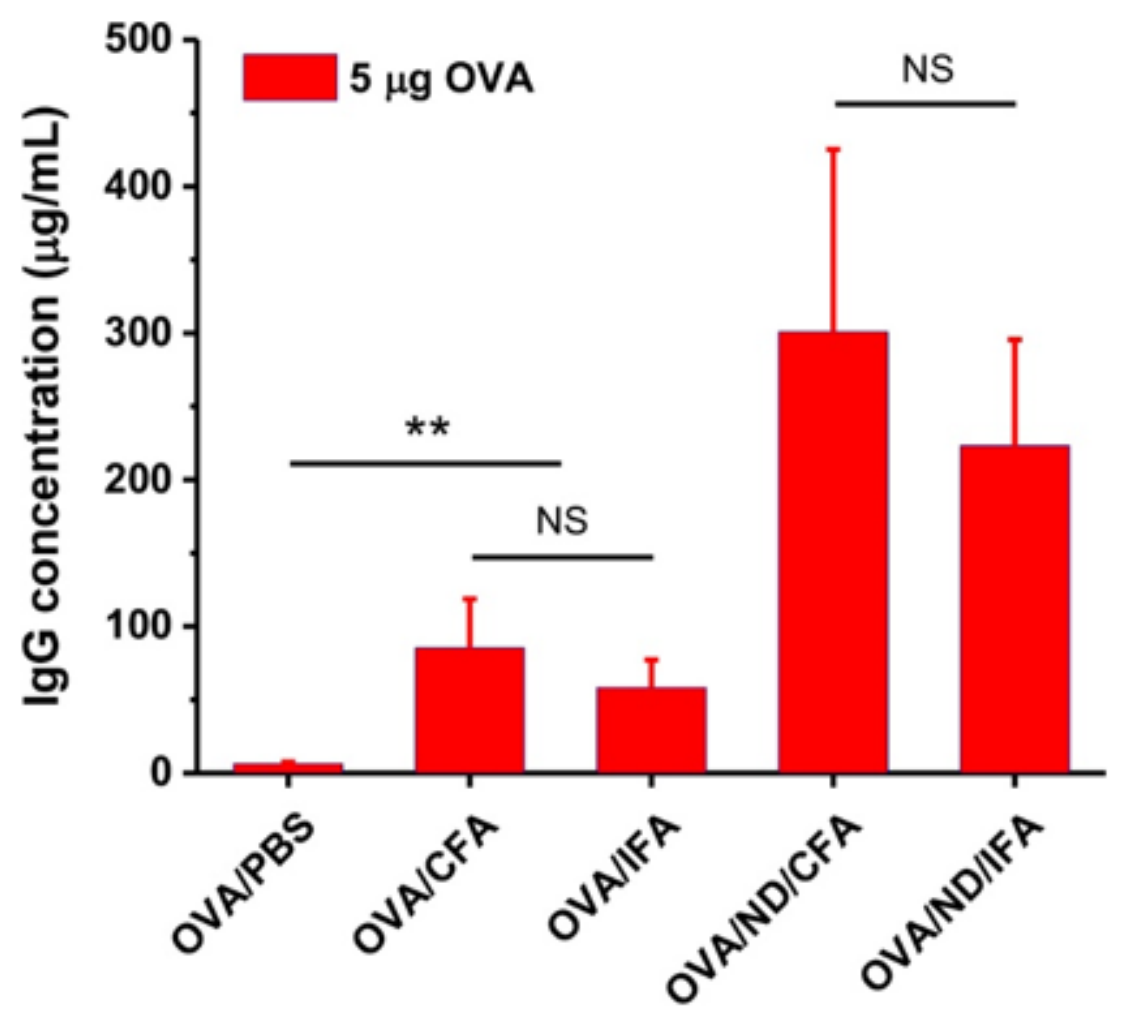

Figure 5

Amount of OVA-specific IgG antibodies produced by the treatments with OVA/ND in IFA or CFA subcutaneously administered into healthy mice. Values are means \pm standard deviations for 3 mice/group (**p $\leq 0.01$, NS: not significant). 


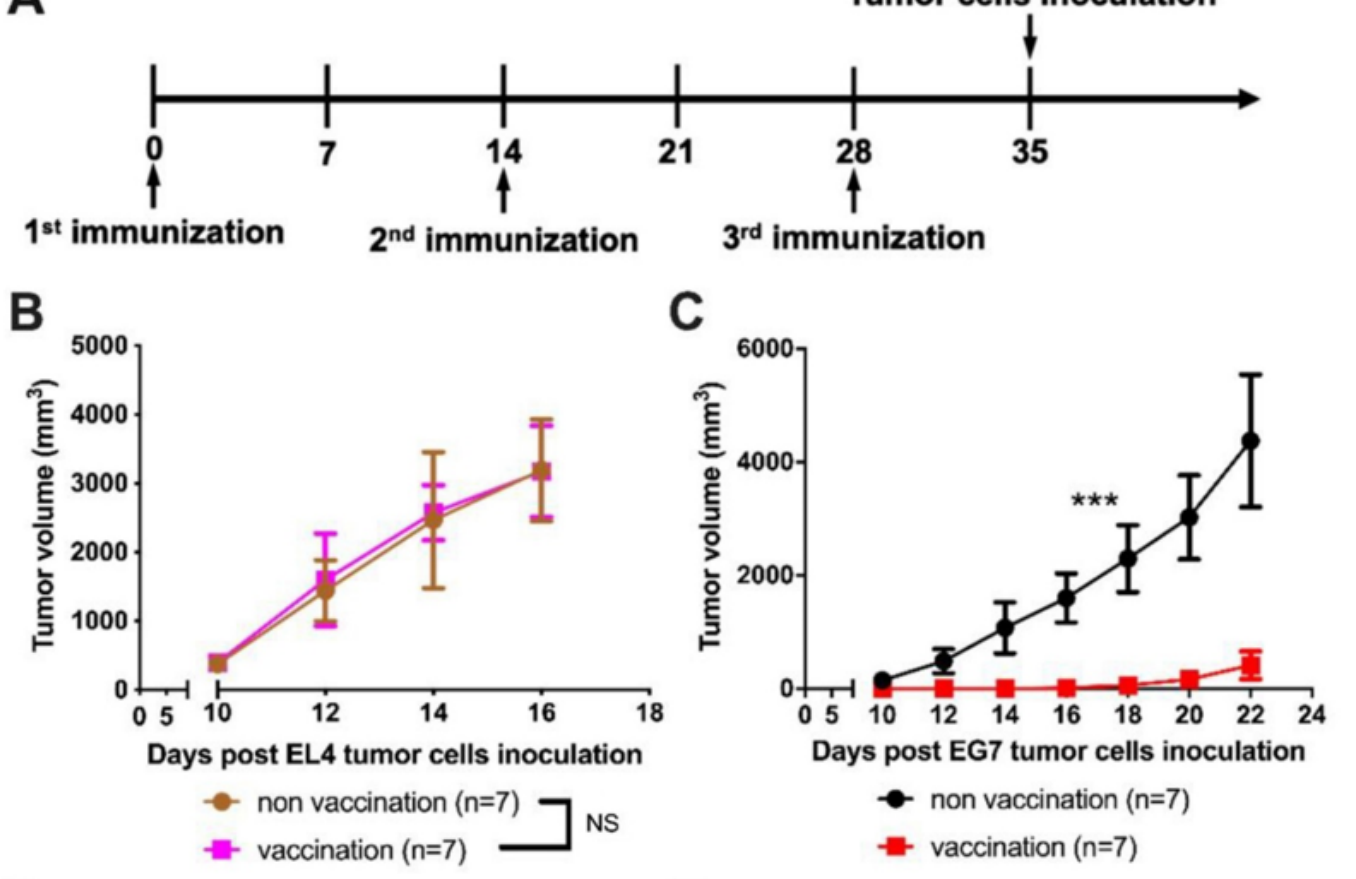

D

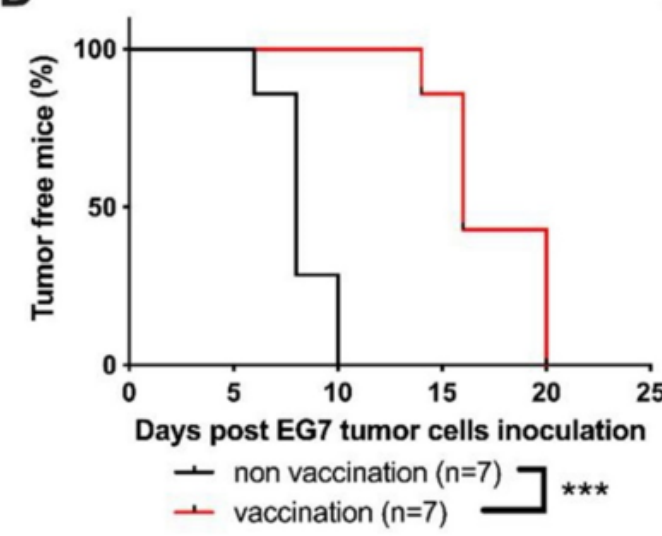

$\mathbf{F}$

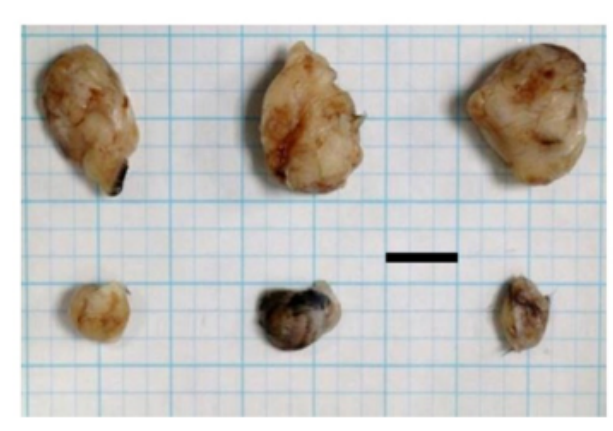

E

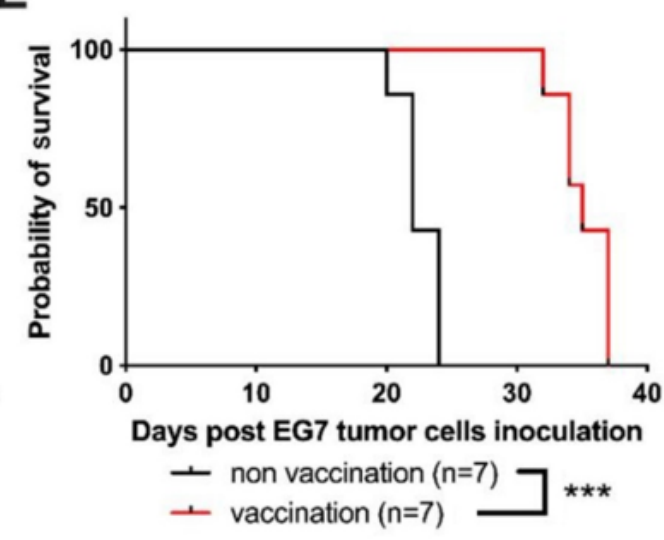

G

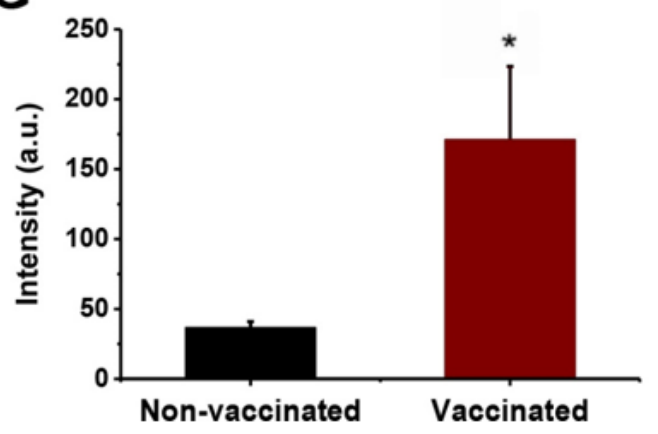

\section{Figure 6}

(A) Timeline of the experimental procedures for using OVA/ND/IFA as antitumor therapeutic agents in mice. (B, C) Changes of the tumor volumes of EL4- (B) or E.G7-OVA- (C) inoculated mice with the tumor sizes measured every 2 days. (D) Comparison of the percentages of the tumor-free mice between nonvaccination groups and vaccination groups in E.G7-OVA-inoculated mice. (E) Comparison of the survival rates between non-vaccination groups and vaccination groups in E.G7-OVA-inoculated mice. (F) 
Comparison of the tumors isolated on day 24 between non-vaccination groups (top) and vaccination groups (bottom) of the E.G7-OVA-inoculated mice. Scale bar: $1.5 \mathrm{~cm}$. Values in (B) - (E) are means \pm standard deviations for 7 mice/group. (G) Quantification of FNDs in spleens isolated on day 24 from nonvaccinated and vaccinated E.G7-OVA-inoculated mice. Values are means \pm standard deviations for 3 mice/group ( ${ }^{\star} p \leq 0.05,{ }^{\star \star \star} p \leq 0.001, \mathrm{NS}$ : not significant).

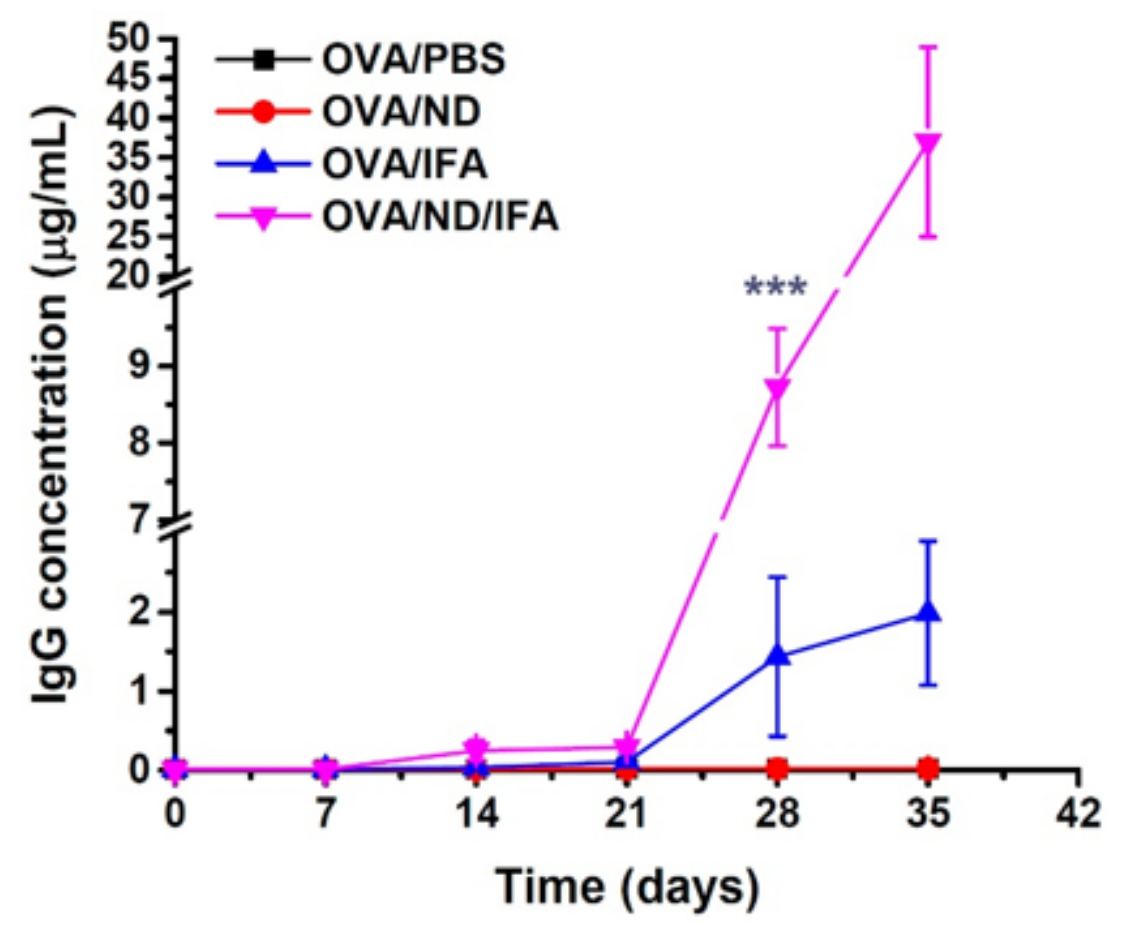

Figure 7

Increases of the concentrations of OVA-specific IgG antibodies in mice subcutaneously administered with four different therapeutic agents. Only one dose of the therapeutic agent was applied in the individual assay. Values are means \pm standard deviations for 3 mice/group ( $\star \star \star p ~ \leq 0.001)$.
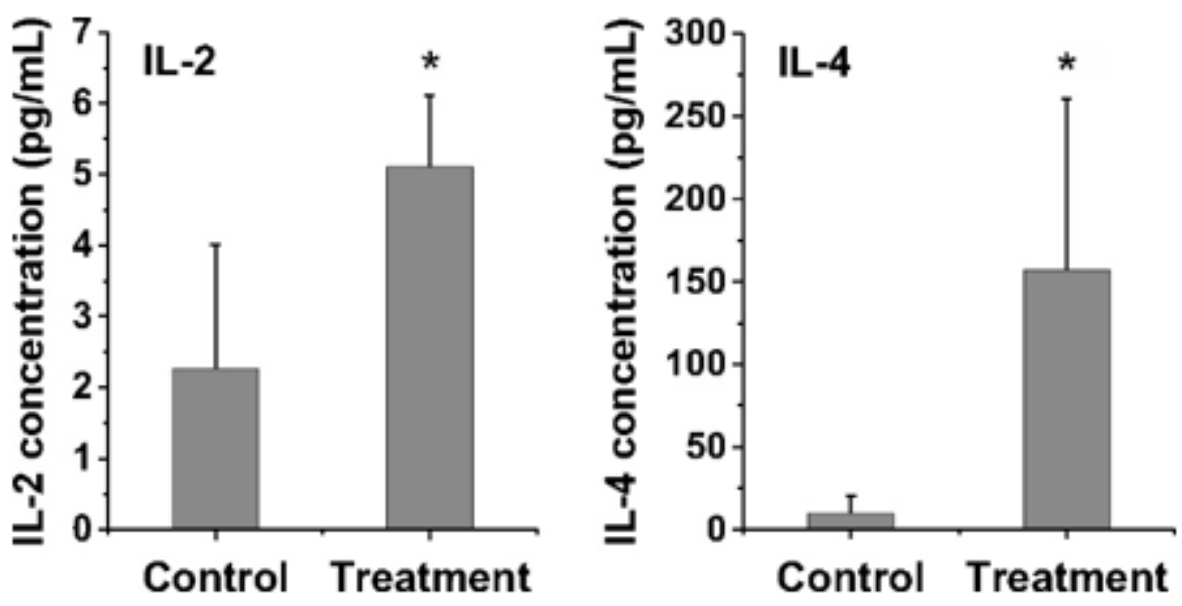

Figure 8 
Changes of IL-2 and IL-4 levels in mice subcutaneously administered with OVA/ND/IFA. The control and treatment groups consist of non-vaccinated and vaccinated mice, respectively. Values are means \pm standard deviations for 3 mice/group ( ${ }^{\star} p \leq 0.05$ ).
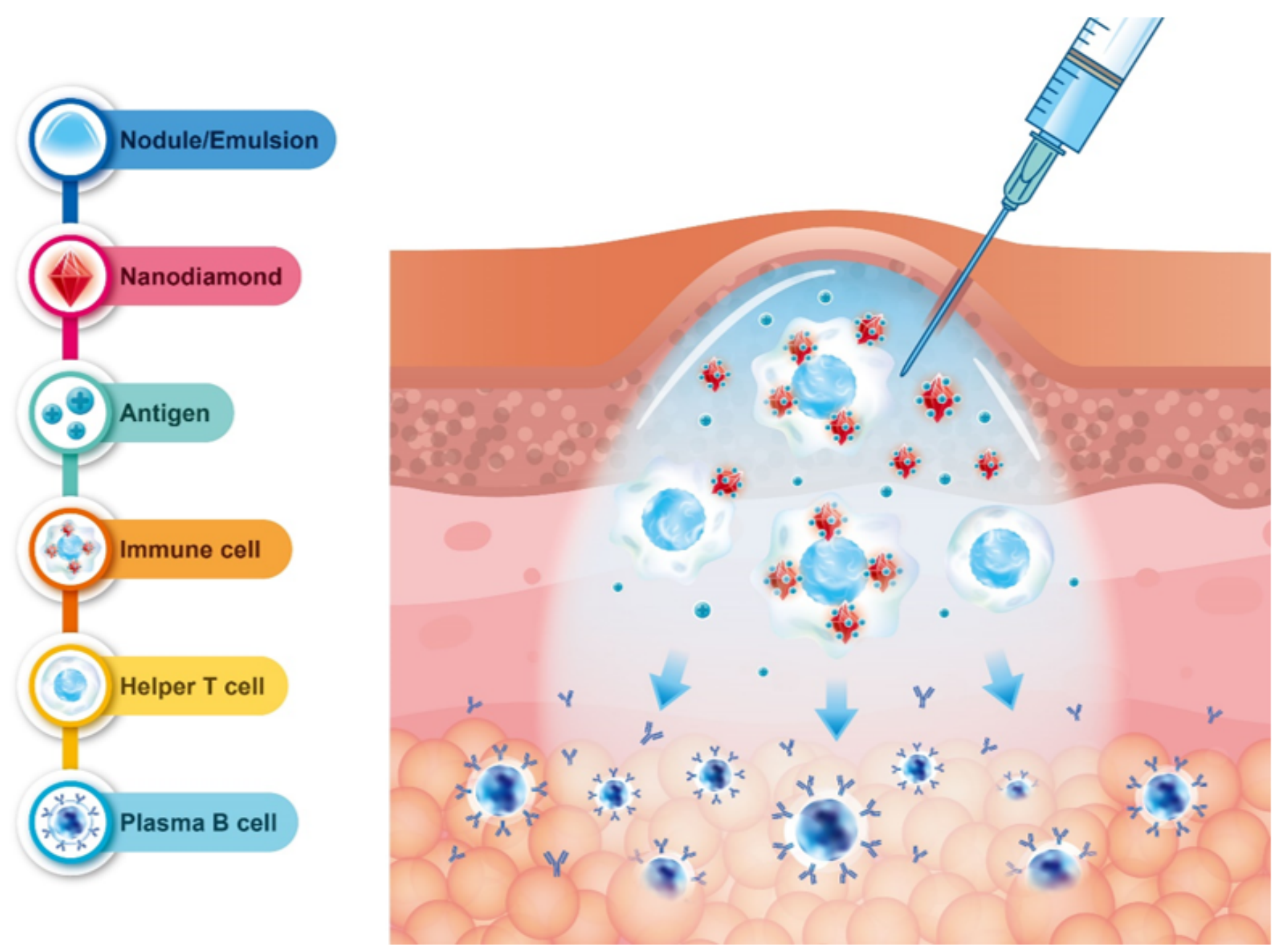

\section{Figure 9}

Proposed predominant mechanism for the induction of immune response by the ND/IFA adjuvants. 